Interviews \& Reviews 



\title{
Arte, Archeologia, Uomo E Natura: Una Conversazione Con Fabio Fogliazza
}

\author{
Matteo Cantisani, School of Archaeology and Ancient History, University of Leicester, UK \\ Fabio Fogliazza, Museo di Storia Naturale di Milano, Italia
}

\begin{abstract}
Introduzione
Fabio Fogliazza è il tecnico per la preparazione di fossili presso la sezione di Paleontologia del Museo di Storia Naturale di Milano, nonché illustratore scientifico ed artista di fama internazionale. Una delle sue opere più famose è la scultura "L'Uomo di Neanderthal", oggi esposta in via permanente al Museo de la Evolución Humana di Burgos (Spagna). In molte sue opere Natura e Uomo vengono riprodotti secondo processi creativi, ponendosi di fatto come piece d'art dalla forte carica emotiva, in grado di suggestionare il pubblico nonostante la distanza geografica e temporale che spesso intercorre tra chi osserva e il soggetto rappresentato. Come redazione siamo entusiasti della possibilità di discutere con Fabio Fogliazza alcune tra queste sue opere in questo numero di Ex Novo, in cui tematiche di management, tutela di beni naturali e conservazione vengono non solo trattate attraverso le lenti scientifiche del sapere ma discutendo anche di approcci in cui emozioni ed esperienze sensoriali vengono incorporate in strategie più complesse. Di seguito si riporta la breve intervista con l'artista Fabio Fogliazza, che ci ha gentilmente permesso di riprodurre in copertina due delle sue opere ed altre ancora di seguito presentate.
\end{abstract}

Matteo Cantisani: Per iniziare, perché non ci racconti un po' di te? La scultura de "l'Uomo di Neandertal", pubblicata sulla copertina del National Geographic Spagna e Portogallo (numero del maggio 2013) è forse tra le opere più famose che hai realizzato ma il tuo percorso artistico è stato lungo.

Fabio Fogliazza: In effetti si. Ho iniziato con la realizzazione di illustrazioni a corredo di pubblicazioni scientifiche: bianco e nero, a china, molto precise ma molto standardizzate... questa tecnica fu il primo passo. All'epoca, quando cominciai nel '92 a collaborare con il Laboratorio di Paleontologia del Museo, il mio lavoro consisteva principalmente in questo e nella preparazione di fossili...sono sempre stato un appassionato di fossili. Solo successivamente si è presentata l'occasione di lavorare a pubblicazioni di più ampio respiro, divulgative e con esse sperimentare nuove tecniche. Mi piacque subito l'acquerello.

\section{MC: Un appassionato di fossili?}

FF: Si, da ragazzo: è stata la passione che mi ha portato a frequentare il Museo di Storia Naturale e la sua biblioteca. Grazie a questa frequentazione, una volta finita l'Accademia di belle Arti di Brera, iniziai a collaborare attivamente con l'Istituto. Il caso ha voluto che proprio allora si era appena concluso il recupero dello scheletro fossile di Besanosaurus (un rettile marino della famiglia degli ittiosauri, lungo circa 6 metri, $\mathrm{NdR}$ ), dallo scavo del Sasso Caldo (VA ) e c'era bisogno di personale per la preparazione: 17.000 ore di preparazione al microscopio. Eravamo tre tecnici, allora. Da cosa nasce cosa e i contratti si sono susseguiti fino a che non sono stato assunto per pubblico concorso nel 2009. 


\section{MC: E la natura in tutto ciò?}

FF: Le rappresentazioni naturalistiche seguirono alle prime esperienze. Un primo avvicinamento a questo tipo di illustrazioni fu attraverso la ricostruzione del contesto ecologico marino del Triassico medio, geomorfologia dei fondali e fauna, sulla base delle evidenze del giacimento di Besano / Monte San Giorgio, oggi patrimonio UNESCO.

\section{MC: Una voglia di raffigurare non solo un soggetto, ma interi mondi scomparsi?}

FF: Certo. Il disegno scientifico è per sua natura un po' didascalico ed è quello normalmente richiesto nell'ambito delle riviste specializzate. L'opportunità di tentare una ricostruzione di ambienti "perduti" è quindi una sfida interessante sotto diversi aspetti: tecnico, interpretativo ma anche da un punto di vista della ricerca in senso stretto. Ho sempre cercato di realizzare immagini apprezzabili esteticamente ma allo stesso tempo scientificamente corrette e che riuscissero a dare un'idea della vita "quando l'uomo non c'era" (citazione di Camille Flammarion): ce l'avrò fatta? Ricostruire questi mondi utilizzando l'acquerello è stato il primo tentativo.

\section{MC: E l'uomo? Che ruolo ricoprono in tutto questo le tue splendide raffigurazioni di} uomini e donne del passato e del presente?

FF: L'uomo è stato sempre, lo è ancora oggi, la mia grande passione. E' stato grazie all'uomo peraltro che ho avuto occasione di sviluppare collaborazioni con diversi specialisti, soprattutto archeologi preistorici, ormai amici, in particolare dell'Università di Ferrara: l'archeologia preistorica è un grande amore, ancora attuale.

\section{MC: Ti riferisci alla già citata scultura "l'Uomo di Neanderthal"?}

FF: Si, in particolare ma non solo. Marco Peresani e la sua equipe avevano concluso da poco la campagna di scavo annuale alla Grotta di Fumane sui monti Lessini in Veneto, uno dei giacimenti più importanti in Italia per quanto riguarda le testimonianze inerenti la transizione Neandertal/Sapiens in Europa. Nel contempo avevano appena pubblicato i risultati delle loro scoperte nei livelli di occupazione musteriani, quelli appunto del Neanderthal. Nacque l'idea di offrire al pubblico e al mondo accademico una rappresentazione basata sulle nuove evidenze, che fosse in grado di esprimere quella componente creativa e simbolica del suo comportamento che fino ad allora era considerata una prerogativa esclusiva di Homo sapiens....mi riferisco al gusto di ornarsi, con tutte le implicazioni sociali e comunicative. Quella scultura è stata in primo luogo un'operazione culturale d'equipe molto ben riuscita: ancora oggi chi volesse riproporre una interpretazione artistica dell'uomo di Neanderthal dovrebbe fare i conti con la nostra.

MC: Dunque si può dire che questo è stato un punto di svolta, se così si può dire, nel tuo percorso di artista e illustratore?

FF: In buona parte sì, ma anche la mostra che Juan Luis Arsuaga mi ha dato l'opportunità di organizzare al Museo de la Evoluciòn Humana (Burgos, Spagna), nel 2014: erano esposte, oltre al modello del Neanderthal, che ora è lì in via permanente, anche alcune mie illustrazioni. Da quel momento mi sono sentito più libero nelle scelte interpretative...i riscontri sono positivi in questo senso. Per fare un esempio: la scelta di disegnare un Neanderthal che gioca a fare l'ombra cinese davanti al focolare (la cosiddetta struttura III di Grotta Fumane) va in questa direzione. Non volevo semplicemente rappresentare l'uomo di Neanderthal seduto 


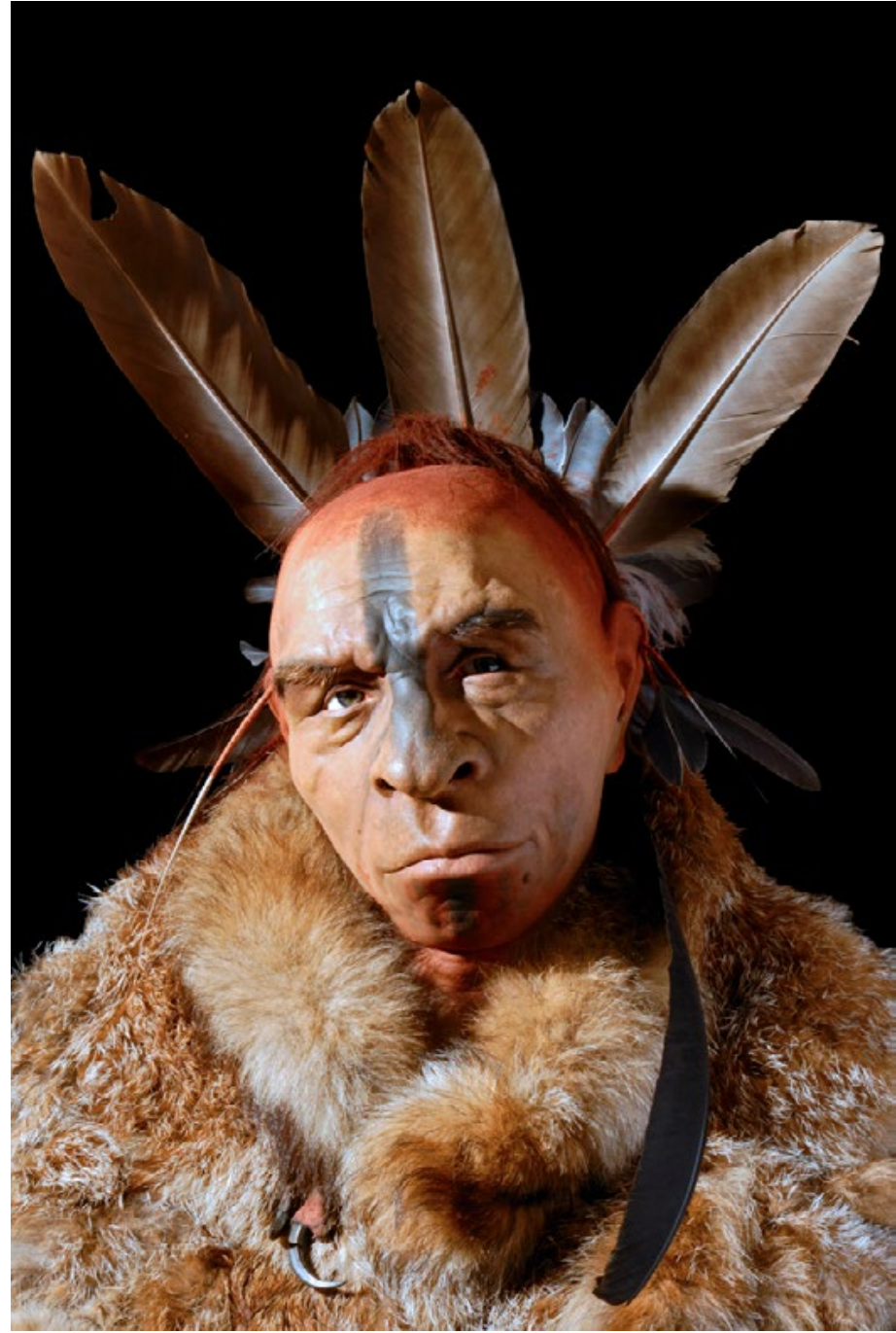

Figura 1: L'Uomo di Neanderthal, scultura in poliuretano (di Fabio Fogliazza (C) vicino al fuoco ma evocarne la modernità. Anche dal punto di vista tecnico mi sono liberato da quei vincoli didascalici che dicevamo prima e ho imposto un mio stile espressivo: può non piacere ma se qualcuno mi chiedesse un'illustrazione, lo farebbe sapendo che quello sarebbe il risultato.

MC: E di "Totem" invece cosa ci puoi dire? In Totem uomo preistorico e natura gli hai rappresentati insieme senza distinguere nettamente un'immagine da un'altra. Come mai?

FF: Appunto, “Totem" è l'esempio perfetto di ciò che ho appena detto. Commissionato dal Museo Archeologico Nazionale di Vicenza: loro sapevano bene cosa aspettarsi ed era proprio quello che volevano in verità. Totem è la colonna stratigrafica, dai recessi della terra arriva al paesino addormentato di oggi. La skyline cittadina in alto rappresenta il top della sequenza stratigrafica. Ma i recessi ci raccontano le storie che in essi sono contenute. La mostra era dedicata al rapporto uomo/orso nella preistoria dei Colli Berici. L’Orso delle Caverne (Ursus spelaeus) era un grande predatore; ancora ai nostri giorni l'orso è visto come una "belva". Nell'illustrazione capovolgo il punto di vista, l'orso diventa la vittima nelle capaci fauci (che sono anche grotta) dell'uomo: un messaggio anche di attualità, se vogliamo, non credi?!. Mi sono letto buona parte della bibliografia scientifica attualmente disponibile sull'argomento, prima di costruire l'impianto illustrativo; per fortuna una consistente fetta di questa era costituita da articoli dell'amico Matteo Romandini (archeozoologo dell'Università di Ferrara), quindi li ho avuti a domicilio in breve tempo.

MC: Questo capovolgimento del punto di vista è molto interessante e sappiamo però che difficilmente etichetti le tue opere come politicamente impegnate ma a questo punto, ed avviandoci verso la conclusione di questa conversazione, la domanda ci sembra d'obbligo: come lo vedi oggi il nostro rapporto con il mondo naturale?

FF: Le mie finalità, intendo professionalmente, non sono quelle di Greta Thunberg; 


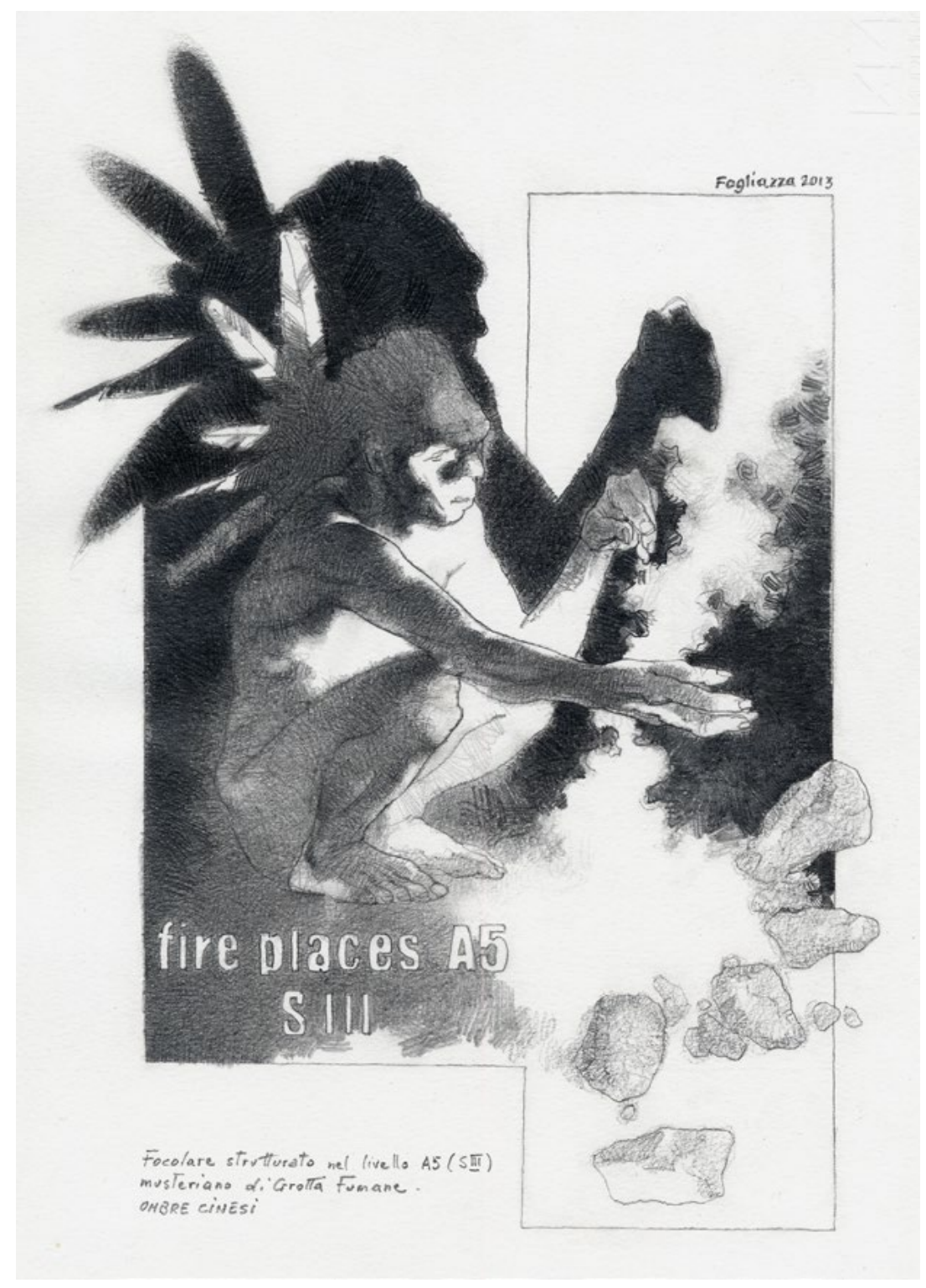

Figura 2: Ombre cinesi (disegno di Fabio Fogliazza (C)

l'approccio ai problemi scientifico/artistici sono altro rispetto all' impegno civico nei confronti dell'ambiente. Non mi sono mai posto la domanda se il pubblico potesse interpretare i miei lavori in senso ecologista; ripeto, non l'ho mai considerato. Chiaramente se così fosse, ne sarei lusingato. Ad ogni buon conto, tutto sommato, credo che siamo dei bravi ragazzi. Gli uomini, intendo: un po' casinisti ma le preoccupazioni verso il nostro bel mondo, le abbiamo. Sono gli interessi economici lo scoglio difficile da superare, soprattutto per chi da quegli interessi ne ricava il proprio benessere e che, guarda caso, possiede anche il potere di veto (devo fare nomi? no dai!) perché agli altri, che sono la maggior parte di noi, per esempio quelli che attraversano il Mediterraneo, probabilmente stanno a cuore altre cose. Sto sembrando un politico, chiudiamo l'inciso.

\section{MC: Grazie mille Fabio, per il tuo tempo.}

FF: Grazie a voi, mi sono divertito. 


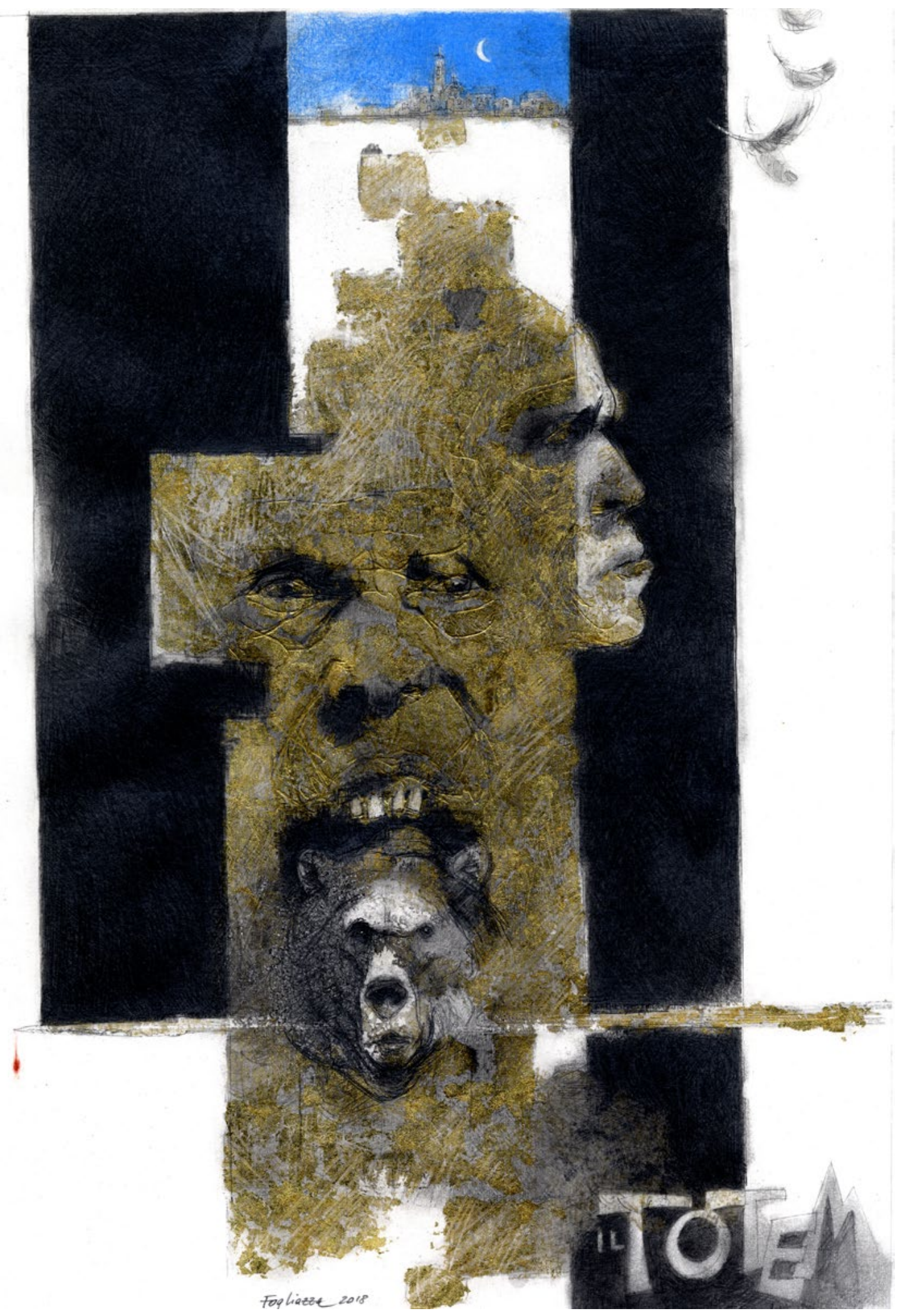

Figura 3: Totem (disegno di Fabio Fogliazza su commissione del Museo Naturalistico Archeologico di Vicenza (C) 